\title{
Flexible regenerative supervision of sequential behaviour
}

\author{
A K RAINA ${ }^{1}$ and A G VALAVI ${ }^{2}$ \\ ${ }^{1}$ Department of Electrical Engineering, Indian Institute of Technology, Kanpur \\ 208 016, India \\ ${ }^{2}$ Wipro Limited (Ist floor), 37 Castle Street, Ashoknagar, Bangalore 560025 , \\ India \\ e-mail: akraina@iitk.ernet.in; valavi@wipinfo.soft.net
}

MS received 14 June 1996; revised 14 May 1997

\begin{abstract}
This paper investigates the non-terminating (NTB) behaviour of discrete-event processes (DEPs) modelled by finite state Büchi automata. Specifically, we develop synthesis criteria and procedures for state-feedback supervisors that ensure infinitely often (i.o.) returns to a given good state subset of the DEP in the closed loop. This problem is referred to as the stabilization problem for discrete event systems (DESs) elsewhere.

The approach of the present paper is distinguished by the fact that the stabilization is achieved without major compromises on the richness of behaviour, or manouverability. This flexibility comes as a consequence of using two supervisors in parallel and switching them on-line to achieve the desired behaviour characteristics in a non-blocking manner. Supervision is arrived at by decomposing the state space into controllable and uncontrollable subspaces relative to the desired target space, much in a manner identical to control theory for systems modelled by.differential equations.
\end{abstract}

Keywords. Regenerative supervision; non-terminating behaviour; discrete event processes.

\section{Introduction}

Supervisory control of discrete-event systems (DESs) modelled as finite-state machines (FSMs) is an important control research area presently. The basic model and control theory, in an FSM-Formal Language set-up, are due to Ramadge and Wonham (Ramadge \& Wonham 1987; Wonham \& Ramadge 1987). Initially, the supervisory theory was developed only for the transient behaviour control of a DES, which behaviour arises, naturally, from simple FSM models and the regular finite-string behaviour sets of such models (Eilenberg 1974; Hopcroft \& Ullman 1979). Later the theory was extended by Ramadge (1989) and others (Kumar et al 1992) to control the steady-state DES behaviour by using Büchii 
extensions of FSMs, or the so-called Büchi automata (Eilenberg 1974), which model nonterminating (or sequential) response of the FSMs.

An important problem that arises in the study of sequential DES behaviour is that of stability. Özveren \& Willsky (1991) define stability as infinitely often (i.o.) guaranteed returns, by means of a state feedback, to a set of $a$ priori selected good states of the DES, in a non-Büchii setting. This paper addresses a similar problem in a Büchii setting which, as mentioned above, arises as the natural extension of FSMs (Eilenberg 1974) to model sequential behaviour. Furthermore, the control strategies developed here ensure flexibility in the sense that the rate of convergence to the good set can be varied on-line, by adjusting a single free parameter, so that depending upon other (possibly unmodelled) conditions one can trade-off richness of behaviour (manouverability) for quick stabilization. This is done by having two logically connected parallel controllers which are switched based on the requirement at any given instant during the operation or evolution of the process. Moreover, the controllers we develop satisfy the required minimal-restrictiveness and non-blocking conditions (Ramadge \& Wonham 1987), so that the closed loop behaviour is maximally-permissible (Wonham \& Ramadge 1987) and satisfies liveness conditions (Özveren \& Willsky 1987). Another important feature is that finite and sequential - that is transient and steady-state - specifications are simultaneously handled, thus simplifying the overall control process. In addition, the stringent requirement of topological closure of the specification behaviour (Ramadge 1989) is avoided here.

There are other notions of stability as well. For example, Brave \& Heyman (1989) consider the good set as an attractive invariant domain in the closed loop under state feedback. The control problem then is to design a feedback so that the DES enters the given set in a finite number of steps and does not exit it later. This could, obviously, constrain the closed loop behaviour seriously since it compromises the richness of behaviour. Other notions of stability that involve the sublanguages of the behaviour of a DES (Kumar et al) rather than state subsets of the underlying FSM also exist. We shall, however, deal only with the state-set defined case, and call our flexible stabilizers regenerative supervisors, to highlight the fact that the controller ensures, in the closed loop, i.o. visitations to the $a$ priori chosen good state subset.

This paper is organised as follows: $\S 2$ develops the necessary formal background for DEPs and their supervisors in terms of both state dynamics and language behaviour. The main section of this paper is $\S 3$, which lays down the synthesis theory for design of the required flexible supervisors. An important part of this section is the proof of the necessity condition of theorem 1 , which is separately given as appendix A due to importance beyond the needs of the theorem. Section 4 concludes the paper by highlighting the advantages of our procedure with the help of a near generic example given in appendix B.

\section{Mathematical preliminaries}

\subsection{Languages}

Let $\Sigma$ be a finite set and $\Sigma^{*}$ the set of all finite strings over $\Sigma$. $\Sigma^{*}$ is a free monoid (Eilenberg 1974) under catenation with the empty string $\epsilon$ being the monoid identity. For $s \in \Sigma^{*},|s|$ 
denotes the length of the string $s$, so that $|\epsilon|=0$. For $u, v \in \Sigma^{*}$, we say that $u$ is a prefix (proper prefix) of $v$, denoted by $u \preceq v(u \prec v)$, if for some $\omega \in \Sigma^{*}, v=u \omega(|\omega|>0)$. Clearly the relation ' $\preceq$ ' is a partial order on $\Sigma^{*}$.

A subset $L \subseteq \Sigma^{*}$ is called a Language over $\Sigma$, and the set $\bar{L}=\{u \mid u v \in L$ for some $v \in$ $\left.\Sigma^{*}\right]$ is called the prefix closure of $L$ (Eilenberg 1974). $L$ itself is prefix closed if $\bar{L}=L$. Next we define $\omega$-Languages (Eilenberg 1974; Ramadge 1989) which constitute infinte or sequential extension of strings.

Let $N$ denote the natural number set and let $\Sigma^{\omega}$ denote the set of sequences over $\Sigma$, i.e.,

$$
\Sigma^{\omega} \doteq\{e \mid e: N \longrightarrow \Sigma\}
$$

For $e \in \Sigma^{\omega}$ and $j \in N$, let $e(j)$ denotes the $j$ th element of $e$, and $e^{j}=e(1) e(2) \ldots e(j)$ denote the finite string (i.e., $e^{j} \in \Sigma^{*}$ ) consisting of first $j$ elements of $e$. In general, $u \in \Sigma^{*}$ will be called a prefix of $e \in \Sigma^{\omega}$ (denoted, once again, by $u \preceq e$ ) if $u=e^{j}$ for some $j \in N . B \subseteq \Sigma^{\omega}$ is called an $\omega$-Language over $\Sigma$, and the language $\operatorname{pr}(B) \in \Sigma^{*}$ defined as;

$$
\operatorname{pr}(B)=\{\epsilon\} \cup\left\{e^{j} \mid e \in B, j \geq 1\right\},
$$

is called the prefix of $B$.

For a monotonic sequence $u_{1} \prec u_{2} \prec u_{3} \cdots$ of elements over $\Sigma^{*}, \exists$ a unique element $e \in \Sigma^{\omega}$ such that $e^{j}=u_{k}$, for $j=\left|u_{k}\right|, k \in N$ (Eilenberg 1974). Such an $e$ is called the limit of the sequence $\left\{u_{k}\right\}$. The adherence or limit of a prefix-closed $L \subseteq \Sigma^{*}$ is the $\omega$-Language $L^{\infty}$ defined as;

$$
L^{\infty}=\left\{e \mid e \in \Sigma^{\omega} \wedge e^{j} \in L, \forall j \in N\right\} .
$$

If $L$ is not prefix closed it suffices that $e^{j} \in L$ for infinitely many $j \in N$ (Eilenberg 1974; Ramadge 1989).

A crucial metric topology on $\Sigma^{\omega}$ is induced by the metric $\rho$, defined as under (Eilenberg 1974);

$$
\begin{aligned}
& \rho\left(e_{1}, e_{2}\right)=1 / n \text { if } e_{1}^{n-1}=e_{2}^{n-1} \text { and } e_{1}^{n} \neq e_{2}^{n} ; \text { and, } \\
& \rho\left(e_{1}, e_{2}\right)=0 \text { if } e_{1}^{n}=e_{2}^{n} .
\end{aligned}
$$

It is easy to show that in this topology $\Sigma^{\omega}$ is compact (Eilenberg 1974). Furthermore, this metric can be extended in a straightforward manner to $\Sigma^{*} \cup \Sigma^{\omega}$, though we shall not need that extension here in the sequel. The topological closure $\bar{B}$ ( $B$ union its limit points) of an $\omega$-Language $B$ equals the limit of its prefix language, i.e., $\bar{B}=[\operatorname{pr}(B)]^{\infty}$, as can be readily verified (Eilenberg 1974; Ramadge 1989). For $B \subseteq S \subseteq \Sigma^{\omega}$, we say $B$ is closed relative to $S$ if $\bar{B} \cap S=B$ (Ramadge 1989).

\subsection{Discrete-event systems}

A discrete-event system (Ramadge \& Wonham 1987) is modelled as a finite state automaton (Eilenberg 1974) $G=\left(Q, \Sigma, \delta, q_{0}\right)$ where $Q$ is the (finite) state set, $\Sigma$ the finite set of events (also called the process alphabet), $\delta: Q \times \Sigma \longrightarrow Q$ gives the state transition (partial) function, which can be naturally extended to $\Sigma^{*}$ by defining 
$\delta(q, \epsilon)=q$, and $\delta(q, s \sigma)=\delta(\delta(q, s), \sigma) \forall s \in \Sigma^{*}$, whenever all intermediate transitions are defined; else it will be undefined. Whenever $\delta(q, s)$ is defined for some $s \in \Sigma^{*}$ we shall denote it by $\delta(q, s)$ !.

We identify the following subsets of $\Sigma$ and $Q$ :

$$
\begin{aligned}
\forall q \in Q ; \Sigma_{q} & :=\{\sigma \in \Sigma: \delta(q, \sigma) !\}, \\
\forall q \in Q ; \forall X \subseteq Q ; \Sigma_{q, X}: & =\{\sigma \in \Sigma: \delta(q, \sigma) \in X\}, \\
\forall X \subseteq Q ; \tau X: & =\{q \in Q: \exists \sigma \in \Sigma \text { s.t. } \delta(q, \sigma) \in X\}
\end{aligned}
$$

$\Sigma_{q}$ defines the active event set at $q$, i.e., set of events executable at $q, \tau X$ is the source set of $X \subseteq Q$, that is the set of states which enter $X$ in a single step and $\Sigma_{q, X} \subseteq \Sigma_{q}$ is the active event subset at $q$ which leads into $X$. For all state subsets $X \subseteq Q$, we shall denote by $\alpha(X)$ the cardinality of $X$ and by $X^{c}$, the complement of $X$. In particular, $\alpha(Q)<\infty$ and $Q^{c}=\Phi$.

For the DES $G$ the finite (transient) behaviour will be given by the prefix closed language $L(G)=\left\{w \mid w \in \Sigma^{*}\right.$ and $\delta\left(q_{0}, w\right)$ ! $\}$. Sometimes, in order to emphasise that this constitutes the event labels of the paths starting at $q_{0}$ of the underlying automaton $G$, we may, instead, call this behaviour $L(G)_{q_{0}}$. We shall also mean by the natural sequential (or, non-terminating) behaviour (NTB) of $G$ the limit $[L(G)]^{\infty}$ which will be assumed to be non-empty throughout. In the simplest form the Büchii extension of $G$ can be defined as follows (Eilenberg 1974; Ramadge 1989). Let $Q_{m} \subseteq Q$ be a given state subset. For each $e \in[L(G)]^{\infty}, \exists$ a unique $S_{e}: N \longrightarrow Q$, such that $S_{e}(j)=\delta\left(q_{0}, e^{j}\right), j \in N$. The state sequence $S_{e}$ is called the state trajectory for the behaviour sequence $e$. The sequence $e$ and the state trajectory $S_{e}$ are said to be admissible, relative to $Q_{m}$ (or, simply admissible) if $S_{e}$ visits the state subset $Q_{m}$ i.o., i.e., if $S_{e}(j) \in Q_{m}$ for infinitely many $j \in N$. The Buichii set $Q_{m}$ then defines the acceptable sequential behaviour of the DES $G$ as follows:

$$
[L(G)]^{\infty} Q_{m}=\left\{e \mid e\left(\in[L(G)]^{\infty}\right) \text { and } S_{e} \text { are admissible }\right\} .
$$

In principle, one can use multiple Büchii (Eilenberg 1974) sets to generate very complex behaviour. In the sequel, however, only one set will be used. Furthermore, it is obvious that $[L(G)]^{\infty}$ itself can be taken as the Büchii behaviour of $G$ for $Q_{m}=Q$.

The control mechanism on DES $G=\left(Q, \Sigma, \delta, q_{0}\right)$ is defined as follows (Ramadge \& Wonham 1989): on $\Sigma$ a partition $\Sigma=\Sigma_{c} \cup \Sigma_{u}$ is defined, where the events in $\Sigma_{c}$ are called the controllable events and those in $\Sigma_{u}$ the uncontrollable events. Let $\Gamma:=\{0,1\}^{\Sigma_{c}}$ be the set of binary assignments on $\Sigma_{c}$, i.e., the discrete topology on $\Sigma_{c}$. Then each $\gamma \in \Gamma,\left(\gamma: \Sigma_{c} \longrightarrow\{0,1\}\right)$ is a control pattern, in the sense that we say $\sigma \in \Sigma_{c}$ is enabled if $\gamma(\sigma)=1$ and is disabled otherwise. Clearly $\gamma \in \Gamma$ can be extended to whole of $\Sigma$ by defining $\gamma(\sigma)=1, \forall \sigma \in \Sigma_{u}$. Given a DES $G=\left(Q, \Sigma, \delta, q_{0}\right)$, the controlled DES $G_{c}:=\left(Q, \Sigma, \delta_{c}, q_{0}\right)$ is defined as follows:

$$
\begin{aligned}
& \delta_{c}: \Gamma \times Q \times \Sigma^{*} \longrightarrow Q \text { s.t. }, \\
&(\gamma, q, \sigma) \mapsto \begin{cases}\delta(q, \sigma), & \text { if }(q, \sigma) ! \text { and } \gamma(\sigma)=1, \\
\text { undefined, } & \text { otherwise }\end{cases}
\end{aligned}
$$

Throughout this paper we shall assume that the $\Sigma$ partition is given and the DES admits the above mentioned control mechanism. We shall, henceforth, drop the subscript $c$ and 
assume $G=\left(Q, \Sigma, \delta, q_{0}\right)$ to be a controlled DES, or a controlled discrete-event process (CDEP).

\subsection{Supervisors for CDEPs}

There are two main supervisory control schemes for CDEPs: (a) dynamic (output) feedback, and (b) state-feedback supervisors.

2.3a Dynamic supervisors: A dynamic supervisor (Ramadge \& Wonham 1987) for a CDEP $G$ is a function $M: L(G) \longrightarrow \Gamma$, which specifies for each string $s \in L(G)$ the subset $\Sigma_{s} \subseteq \Sigma$ of enabled events $\left(\Sigma_{s}:=\{\sigma: M(s) \sigma=1\}\right)$. The closed-loop behaviour of $G$ supervised by $M$, denoted by $L(G / M)$, is given by:

$$
\begin{aligned}
& \epsilon \in L(G / M), \text { and } \\
& \omega \sigma \in L(G / M) \Leftrightarrow \begin{cases}(i) & \omega \in L(G / M), \\
\text { (ii) } & M(\omega) \sigma=1, \text { and } \\
\text { (iii) } & \omega \sigma \in L(G) .\end{cases}
\end{aligned}
$$

The non-terminating (or, steady-state) behaviour of $G$ supervised by $M$ is, obviously, then given by $[L(G / M)]^{\infty}$.

2.3b State-feedback supervisors: More important, from the point of view of applicability and design simplicity, is the state-feedback supervisor (Kumar et al 1993a) which, for a given CDEP $G$ is defined as a function $R: Q \rightarrow \Gamma$. Such a supervisor specifies, for each state of CDEP, the set of enabled events. The closed-loop system, where $G$ is supervised by $R$, can be viewed as another state machine $(G / R):=\left(Q, \Sigma, \delta_{R}, q_{0}\right)$, where $\delta_{R}(q, \sigma)=\delta(q, \sigma)$ if $R(q) \sigma=1$, and, $\delta_{R}(q, \sigma)$ is undefined if $R(q) \sigma=0$. The non-terminating behaviour of the closed-loop is, once again, given by $[L(G / R)]^{\infty}$.

An important property that any supervisor should satisfy is that every response sequence begun (in the closed-loop situation) must be allowed to reach a steady state; in other words, every finite string generated by the closed loop must lead to a non-terminating behaviour sequence. This is called the property of non-blocking or the liveness property of the system which implies that the system does not stall along a response trajectory. Formally, a supervisor $M$ (dynamic or static) for a CDEP $G$ is said to be non-blocking if:

$$
\forall s \in L(G / M) ; \exists e \in[L(G / M)]^{\infty} \text { s.t. } s=e^{j} \text { for some } j \in N .
$$

Given a CDEP $G$, and an $X \subseteq Q$, a supervisor $M$ is said to be $X$-invariant if:

$$
\forall s \in L(G / M), \delta\left(q_{0}, s\right) \in X
$$

An $X$-invariant (or, equivalently, a state-invariant) supervisor will, therefore, restrict the system behaviour to a particular state subset in the closed loop. All transitions to outside of the pre-specified set are disabled. Note that if $X_{a}$ is the set of closed-loop accessible states then $X_{a} \subseteq X$. 
2.3c Regenerative supervisors: Given the CDEP $G$ and a state subset $Q_{m} \subseteq Q$, a supervisor $M$ is said to be $Q_{m}$-regenrative if:

$$
\begin{aligned}
& {[L(G / M)]^{\infty} \neq \Phi, \text { and }} \\
& {[L(G / M)]^{\infty} \subseteq[L(G)]^{\infty} Q_{m} .}
\end{aligned}
$$

The supervisor $M$ for $G$ is such that the NTB is non-empty and the set $Q_{m}$ is visited i.o. Note that this does not imply that the only states visited i.o. are the $Q_{m}$ states. Also note that this is identical, in principle, to the stability notion of Özveren \& Willsky (1991), as introduced earlier, although the setting here is Büchii in contrast, wherein the sequential behaviour is defined in terms of the admissibility conditions as given in (9).

The problem addressed to in the sequel is the following:

Given a CDEP $G=\left(Q, \Sigma, \delta, q_{0}\right)$ and the partition $\Sigma=\Sigma_{u} \cup \Sigma_{c}$, and a state subset $Q_{m} \subseteq Q$

- Determine if there exists a supervisor $M$ for $G$ that is both non-blocking and $Q_{m^{-}}$ regenerative. (Henceforth, we shall simply call these supervisors regenerative once the set $Q_{m}$ is understood as identified).

- If $M_{\alpha}$ is the non-empty family of non-blocking regenerative supervisors then find $M \in$ $M_{\alpha}$ such that:

$$
[L(G / S)]^{\infty} \subseteq[L(G / M)]^{\infty} \forall S \in M_{\alpha},
$$

i.e., find the minimally restrictive of all the non-blocking supervisors for $G$.

We shall call these supervisors simply regenerative whenever the Büchii set $Q_{m}$ is specified or obvious.

\section{Main results}

In this section we develop a controller synthesis for solving the above mentioned stabilization problem. The synthesis procedure is based on construction of a series of state subsets, which are recursively reachable and within the union of which the state invariance of a supervisor can be easily achieved.

\subsection{Control subsets}

Given $G=\left(Q, \Sigma, \delta, q_{0}\right), \Sigma=\Sigma_{u} \cup \Sigma_{c}$, and $Q_{m}(\neq \Phi) \subseteq Q$, define the state subset sequences $Q_{i}$ and $X_{i}$ as follows:

$$
\begin{aligned}
Q_{0} & \doteq X_{0} \doteq Q_{m}, \\
X_{i+1} & \doteq\left\{q \in\left(Q_{i}^{c} \cap \tau Q_{i}\right): \Sigma_{\left.q, Q_{i}^{c} \subseteq \Sigma_{c}\right\},}\right. \\
Q_{i+1} & \doteq Q_{i} \cup X_{i+1} .
\end{aligned}
$$

Note that $X_{1}$, for example, is that subset of $Q_{0}^{c}$ which can be driven to $Q_{0}$ in a single transition. This is because there is a transition to $Q_{0}$, and all transitions to $Q_{0}^{c}$ are controllable 
(and, therefore, can be disabled). Since $\left\{Q_{i}\right\}$ is an ascending chain we define, First Control Subset (FCS) $\hat{Q}$ as its limit, given by:

$$
\hat{Q} \doteq \sup _{i} Q_{i} \doteq \bigcup_{i} Q_{i} \doteq \bigcup_{i} X_{i}
$$

The FCS $\hat{Q}$ is, therefore, the largest state subset which reaches $Q_{m}$, possibly uncontrollably, but whose dynamics outside of itself, being controllable, can be disabled. To control escape dynamics from $Q_{m}$ itself, we define, First Regenerative Set (FRS) $\hat{Q}_{m}$ as:

$$
\hat{Q}_{m} \doteq\left\{q \in\left(Q_{m} \cap \tau \hat{Q}\right): \Sigma_{q, \hat{Q}^{c}} \subseteq \Sigma_{c}\right\} .
$$

The state set $\hat{Q}_{m}$ is clearly the Büchii subset which can be made invariant in the closed loop by disabling the exit transitions.

Some interesting properties of the sets $X_{i}, \hat{Q}$ and $\hat{Q}_{m}$, which essentially identify controllable transitions at various levels, are listed below (see Valavi for more properties and proofs):

$$
\begin{aligned}
& \exists k \leq \alpha\left(Q_{m}^{c}\right) \text { s.t. } X_{i}=\Phi \quad \forall i>k, \text { and } ; \\
& \forall i \leq k, X_{i} \neq \Phi, \text { and } \\
& Q_{k}=\hat{Q}=\bigcup_{i=0}^{k} X_{i} . \\
& \forall i \geq 1, \forall q \in X_{i}, \bigcup_{j \geq i} \Sigma_{q, X_{j}} \subseteq \Sigma_{c} . \\
& \forall q \in \hat{Q}-Q_{0}, \Sigma_{q, \hat{Q}^{c}} \subseteq \Sigma_{c} . \\
& \forall i \geq 1, \forall q \in X_{i}, \bigcup_{j=0}^{i-1} \Sigma_{q, X_{j}} \neq \Phi .
\end{aligned}
$$

Intuitively, these properties all stem from the structure of the $X_{i}$ chain, which is such that all dynamics from the lower to higher index can be disabled. Therefore, as the first approximation, the supremum of the chain contains all the states that can be driven to $Q_{m}$ either uncontrollably, or by enabling all controllable events along the path. Also note that property 21 is valid for a finite $Q$, whereas others hold independently of its cardinality. We shall, however, consider our CDEPs to be finite state only, as earlier mentioned, for practical reasons, even if some of the results hold in general.

Next, we develop the regenerative supervisors for our problem as defined in \$2.3. This will be achieved by synthesis of a $\hat{Q}$-invariant supervisor, so that all potential paths that escape $Q_{m}$ indefinitely are disabled. As will be clear from the sequel, it is always possible to do so under the fairly mild condition that $q_{0}$ be in $\hat{Q}$, i.e., in one of the $X_{i}$ 's, which are state sets that can always be driven to $Q_{m}$ in a finite number of steps. Initially, to to keep the synthesis simple and tractable, we shall assume that the FRS $\hat{Q}_{m}$ equals $Q_{m}$. Later this condition will be relaxed.

Note that $\forall q \in Q_{m}-\hat{Q}_{m}$; either $\Sigma_{q, \hat{Q}}=\Phi$ or $\Sigma_{q, \hat{Q}^{c}}$ is uncontrollable. For regenerative behaviour, it should be possible to disable transitions to $\hat{Q}^{c}$, and for non-blocking condition 
there should be a transition to $\hat{Q}$. Therefore, $Q_{m}-\hat{Q}_{m}$ is the set of states that violates one or both of these conditions. After the supervisory syntheses given below this problem will be addressed to, so that the final synthesis is free of this apparently severe constraint that $Q_{m}$ equal $\hat{Q}_{m}$.

Theorem 1. [(The R-supervisor)] Given the CDEP $G=\left(Q, \Sigma, \delta, q_{0}\right)$, with $\Sigma=\Sigma_{u} \cup$ $\Sigma_{c}, Q_{m} \subseteq Q$, the FCS $\hat{Q}$ and the FRS $\hat{Q}_{m}$, assume that $\hat{Q}_{m}=Q_{m}$. Then there exists $a$ non-blocking and regenerative supervisor $R$ for $G$ if and only if $q_{0} \in \hat{Q}$.

Proof. Sufficiency: Let $q_{0} \in \hat{Q}$. Then from the above properties we know that $\hat{Q}=$ $\bigcup_{i=0}^{k} X_{i}$ for some $k \leq \alpha\left(Q_{m}^{c}\right) \leq \alpha(Q)$ and $X_{i} \neq \Phi, 0 \leq i \leq k$.

Define a state feedback supervisor $R$ as follows:

$$
\begin{aligned}
\forall q \in Q_{0}, R(q) \sigma=0 \forall \sigma \in \Sigma_{q, \hat{Q}^{c}} \\
\\
\quad\left[\subseteq \Sigma_{c} ;(20)\right], \\
\forall i \geq 1, \forall q \in X_{i}, R(q) \sigma=0 \forall \sigma \in\left(\bigcup_{j \geq i}^{k} \Sigma_{q, X_{j}}\right) \cup\left(\Sigma_{q, \hat{Q}^{c}}\right), \\
\\
{\left[\subseteq \Sigma_{c} ; \text { properties } 21,23\right] } \\
R(q) \sigma=1, \text { otherwise. }
\end{aligned}
$$

$R$, as is obvious, is a supervisor that drives the system fastest to $Q_{m}$, the desired state set for regenerative operation, by preventing escape from $\hat{Q}$ as well as any backward movement (towards increasing index on $Q_{i}$ ) within $\hat{Q}$. Therefore, once the system is in $\hat{Q}$, it will move into $Q_{m}$ in at most $k$ steps.

The closed loop system $G / R \doteq\left(Q, \Sigma, \delta_{R}, q_{0}\right)$, is then defined by:

$$
\delta_{R}(q, \sigma)= \begin{cases}\delta(q, \sigma), & \text { if } R(q) \sigma=1, \text { and } \\ \text { undefined, } & \text { if } R(q) \sigma=0\end{cases}
$$

To show that $R$ is non-blocking, note that $\forall q \in \hat{Q}, \delta_{R}(q, \sigma)$ ! (is defined) $\Longrightarrow \delta_{R}(q, \sigma) \in$ $\hat{Q}$, because transitions from $\hat{Q}$ to its complement are disabled by the supervisor $R$ ((25), (26)). Therefore, it follows that:

$$
q_{0} \in \hat{Q} \Longrightarrow \delta_{R}\left(q_{0}, s\right) \in \hat{Q}, \forall s \in L(G / R) .
$$

In other words, $R$ is a $\hat{Q}$-invariant supervisor. Furthermore, (20), (24), (27) and the fact that $\hat{Q}=Q_{0} \cup\left(\bigcup_{i=1}^{k} X_{i}\right)$ together imply that:

$$
\forall q \in \hat{Q} ;\left\{\sigma \in \Sigma_{q, \hat{Q}}: R(q) \sigma=1\right\} \neq \Phi .
$$

Next, from (29) and (30), it follows that $\forall s \in L(G / R), \exists \sigma \in \Sigma$ such that $s \sigma \in L(G / R)$. Therefore, $\forall s_{0} \in L(G / R), \exists$ a sequence $s_{0} \prec s_{1} \prec s_{2} \cdots$ in $L(G / R)$, so that $R$ is a non-blocking supervisor that keeps the closed-loop live.

To show that $R$ is regenerative, it suffices to show that a transition to $X_{0}\left(=Q_{m}\right)$ is guaranteed in at most $k$ steps. To this end assume that $s, s \omega \in L(G / R)$, with $\delta_{R}\left(q_{0}, s\right)=$ $q \in X_{r}$, for some $1 \leq r \leq k$ (this is guaranteed by $q_{0} \in \hat{Q}$ and the supervisor design (25)-(27), and $|\omega| \geq k$. Let $\sigma_{k-1} \sigma_{k-2} \cdots \sigma_{0}$ be the $k$-long prefix of $\omega$ (note the reverse index on $\omega$ ). Clearly $\bar{q}_{j}=\delta_{R}\left(\bar{q}_{j-1}, \sigma_{k-j}\right) \in X_{r_{j}}$, where $\bar{q}_{0}=q$ and $r>r_{1}>r_{2} \cdots$. 
Since the index $r_{i}$ decreases at least as fast as the index on $\sigma$, we have $q_{i} \in X_{0}$ for some $i \leq k$. Furthermore, if $q \in X_{0}, \delta_{R}\left(q, \sigma_{k-1}\right) \in X_{0} \cup X_{r}$, for some $1 \leq r \leq k$, the same process holds. Thus, in the closed loop a transition to $Q_{m}\left(=X_{0}\right)$ is always guaranteed (in $\leq k$ steps) and, therefore, the supervisor is regenerative.

Necessity. See appendix A.

Remark 1. It is obvious from appendix A that regenerative and non-blocking supervisors are $\hat{Q}$-invariant, since for every sub-CDEP that evolves from a reachable state $q \in Q$, we have that $q \in Q_{i}$ (for some $\left.i\right) \subseteq \hat{Q}$. This implies that only the states from $\hat{Q}$ can be regeneratively driven to $Q_{m}$ along non-blocking trajectories.

3.1a Minimally restrictive supervisors: The $R$-supervisor, though non-blocking and regenerative is, very restrictive in terms of the richness of the closed-loop behaviour. In fact, as is obvious, the $R$-action is to select control patterns to drive the closed-loop to $Q_{m}$ as fast as possible. This constrains the behaviour profile of the system. To overcome this problem we use another supervisor in parallel with the $R$-supervisor, so that the closed-loop is both rich in behaviour (minimally restrictive) as well as regenerative.

Define a state-feedback supervisor $S: Q \longrightarrow \Gamma$ for the $\operatorname{CDEP} G$ as:

$$
\begin{gathered}
\forall q \in \hat{Q} ; \quad S(q) \sigma=0 \forall \sigma \in \Sigma_{q, \hat{Q}^{c}}\left[\subseteq \Sigma_{c}\right], \\
S(q) \sigma=1 \text { otherwise. }
\end{gathered}
$$

If $q_{0} \in \hat{Q}$ the supervisor $S$ is $\hat{Q}$-invariant, since $\forall q \in \hat{Q}$ the transitions that lead to a state in $\hat{Q}^{c}$ are disabled. Note that the supervisor $S$, while ensuring that the system remain inside $\hat{Q}$, does not drive it hard to $Q_{m}$, the way $R$ does. We now have the following straightforward result.

Theorem 2. Let $G$ be a CDEP, as in theorem 1, with $\hat{Q}_{m}=Q_{m}$, and $q_{0} \in \hat{Q}$. Let $S$ be the state feedback supervisor for $G$ as defined above. Then, if $M$ is any non-blocking and regenerative supervisor for $G$, we have that:

$$
L(G / M) \subseteq L(G / S) .
$$

In other words, $S$ is the minimally restrictive $\hat{Q}$-invariant supervisor, richer in behaviour than the non-blocking and regenerative supervisor subclass.

Proof. (Straightforward) We know that every non-blocking and regenerative supervisor is $\hat{Q}$-invariant under the given conditions (see remark 1). Therefore, since $q_{0} \in \hat{Q}$ and the states in $\hat{Q}^{c}$ cannot be driven to $Q_{m}$ in the desired manner, every regenerative and non-blocking supervisor must disable transitions to $\hat{Q}^{c}$.

Therefore, $s \in L(G / M) \Longrightarrow \delta\left(q_{0}, \omega\right) \in \hat{Q}, \forall \omega \in \operatorname{pr}(s)$. Also since the only transitions that $S$ disables are those to $\hat{Q}^{c}$, we have that $\delta\left(q_{0}, \omega\right) \in \hat{Q} \Longrightarrow \omega \in L(G / S), \forall \omega \in$ $\operatorname{pr}(s) \Longrightarrow s \in L(G / S)$. The empty string $\epsilon$ in $\operatorname{pr}(s)$ does not affect the argument since $q_{0} \in \hat{Q}$. This completes the proof. 


\subsection{Flexible supervisors}

From the preceeding discussion it is obvious that the $R$-supervisor capability of fastest motion to the desired state set constrains its behaviour, whereas, in contradistinction, the $S$-supervisor's minimally restrictive behaviour limits its frquency of arrival into the desired state set. In practice, a compromise is desirable. In the rest of this section we shall address this problem and provide a solution based on continual switching between $R$ and $S$ supervisors that, dynamically, takes into account the last arrival into $Q_{m}$.

First we define a delay function $g: L(G) \longrightarrow N \cup\{0\}$ ( $\mathrm{N}$ is the set of natural numbers) as:

$$
\begin{aligned}
g(\epsilon) & =0, \\
g(s \sigma) & = \begin{cases}g(s)+1 & \text { if } \delta\left(q_{0}, s \sigma\right) \in Q_{m}^{c}, \text { and } \\
0 & \text { if } \delta\left(q_{0}, s \sigma\right) \in Q_{m} .\end{cases}
\end{aligned}
$$

The delay function is aperiodically cyclic and in every cycle counts the number of events executed outside of $Q_{m}$, so that a large value in any cycle reflects large behaviour executed without returning to $Q_{m}$. Next, given a $k \in N$, we define a k-jump function $f_{\mathbf{k}}: N \cup\{0\} \longrightarrow\{0,1\}$ as:

$$
f_{\mathbf{k}}(i)= \begin{cases}0, & \text { if } i<k, \text { and } \\ 1, & \text { if } i \geq k\end{cases}
$$

The composite sequence $L(G) \stackrel{g}{\longrightarrow} N \cup\{0\} \stackrel{f_{k}}{\longrightarrow}\{0,1\}$, represented by the function composition $f_{\mathbf{k}} \circ g$, therefore, becomes high every time the CDEP executes more than $\mathbf{k}$ events in a row outside $Q_{m}$. The composite function $f_{\mathbf{k}} \circ g$ will henceforth be called the system switching function of order $k$.

The switching function is used for control strategy as follows: Run the CDEP $G$ under the supervisor $S$ as long as the switching function is low $(=0)$ and once the switching function becomes high $(=1)$ the supervisor $R$ takes over. The parameter $k$ can be dynamically varied to satisfy the requirement on the rate of visitations to $Q_{m}$. Note that $k=0 \mathrm{implies}$ running the system only under $R$, since the fastest convergence to $Q_{m}$ is desired and as $k \rightarrow \infty$ only $S$ will be used since the constraint now is the richness of behaviour rather than fast settling to $Q_{m}$. The composite overall dynamic supervisor (see 2.3a) $T$ modelling the abovementioned control strategy can be defined as under.

DEFINITION. Given a CDEP $G=\left(Q, \Sigma, \delta, q_{0}\right), \Sigma=\Sigma_{u} \cup \Sigma_{c}$ and the associated state sets $Q_{m}, \hat{Q}$, and $\hat{Q}_{m}$ such that $\hat{Q}_{m}=Q_{m}$ and $q_{0} \in \hat{Q}$. Let $k \in N \cup\{0\}$ be given and the supervisors $R$ and $S$ be as defined earlier. Further let the switching function $f_{\mathbf{k}} \circ g$ be as defined above.

Define the dynamic supervisor $T: L(G) \longrightarrow \Gamma$ as:

$$
T(s) \sigma=\left\{\left[f_{\mathbf{k}} \circ g(s)\right] \wedge\left[R\left(\delta\left(q_{0}, s\right)\right) \sigma\right]\right\} \vee\left\{\neg\left[f_{\mathbf{k}} \circ g(s)\right] \wedge\left[S\left(\delta\left(q_{0}, s\right)\right) \sigma\right]\right\} .
$$

From the preceeding discussion it follows that for finite $k$ the supervisor $T$ is both non-blocking and regenerative with the richness of behaviour increasing as $k$ becomes 
larger. It should be noted that the parameter $k$ can be dynamically varied on-line to suit any specification changes or needs of the environment.

The computational effort involved in $T$ synthesis is mainly in the recursive computation of the sets $X_{i}$, so that a loose (conservative) upper bound on the complexity is $\bigcirc\left(n^{2} p\right)$, where $n=\alpha(Q)$ and $p$ is the number of edges of $G$.

\subsection{Weak supervisory conditions}

As explained in $\$ 3.1$, for regenerative behaviour it should be possible to disable transitions to $\hat{Q}^{c}$ and for non-blocking behaviour transitions to $\hat{Q}$ must be ensured. The states in $Q_{m}-\hat{Q}_{m}$ are precisely those Büchii states that violate one or both of these conditions. Therefore, for desired behaviour it must be ensured that these states are kept out of the possible closed-loop paths with minimal reduction in the desired behaviour set.

This can be done as follows; Let $G$ be given such that $\hat{Q}_{m} \neq Q_{m}$. We proceed to build a family of control subsets as follows.

Let $Q_{m_{0}} \doteq Q_{m} ; \hat{Q}_{0} \doteq \hat{Q} ; \hat{Q}_{m_{0}} \doteq \hat{Q}_{m}$. Define:

$$
\begin{aligned}
Q_{m_{i}} & =\hat{Q}_{m_{i-1}}, \\
\hat{Q}_{i} & =\text { set obtained from FCS construction with } Q_{m_{i}} \text { as } Q_{m}, \\
\hat{Q}_{m_{i}} & =\text { set obtained from eqn. } 20 \text { with } Q_{m_{i}} \text { as } Q_{m} \text { and } \hat{Q}_{i} \text { as } \hat{Q}
\end{aligned}
$$

Note that the chains $Q_{m_{i}}, \hat{Q}_{i}$ and $\hat{Q}_{m_{i}}$ are all descending. Next define:

$$
\begin{gathered}
\hat{Q}_{s} \doteq \inf _{i} \hat{Q}_{i} \doteq \bigcap_{i} \hat{Q}_{i}, \\
Q_{m_{s}} \doteq \inf _{i} Q_{m_{i}} \doteq \bigcap_{i} Q_{m_{i}}, \\
\hat{Q}_{m_{s}} \doteq \inf _{i} Q_{m_{i}} \doteq \bigcap_{i} \hat{Q}_{m_{i}} .
\end{gathered}
$$

The role played by $\hat{Q}_{s}$ and $\hat{Q}_{m_{s}}$ is the same as that of $\hat{Q}$ and $\hat{Q}_{m}$, respectively, in the earlier case when $\hat{Q}$ was assumed equal to $\hat{Q}_{m}$. In other words, transitions to $\hat{Q}_{m_{s}}$ can always be guaranteed from $\hat{Q}_{s}$, whereas from outside of $\hat{Q}_{s}$ this cannot be achieved. Therefore, we shall call $\hat{Q}_{s}$ the Infimal control subset (ICS) and $\hat{Q}_{m_{s}}$ the Infimal regenerative subset (IRS). It is easily verified that $\hat{Q}_{m_{s}}=Q_{m_{s}}$. We call the set $Q_{m_{s}}$ the Infimal marked subset (IMS). It is obvious that if the algorithm of theorem 1 is run using $\hat{Q}_{m}$ as $Q_{m}, \hat{Q}_{s}$ as $\hat{Q}$ and $\hat{Q}_{m_{s}}$ as $\hat{Q}_{m}$, then the following generalization of that theorem holds.

Theorem 3. (Generalization) Given a $C D E P G=\left(Q, \Sigma, \delta, q_{0}\right)$, with $\Sigma=\Sigma_{u} \cup \Sigma_{c}$, and the Büchii state subset $Q_{m} \subseteq Q$, let $\hat{Q}_{s}$ and $\hat{Q}_{m_{s}}$ be the associated ICS and IRS, respectively. Then $G$ is a regenerative $C D E P$ (i.e., there exists a regenerative supervisor for $G$ ) if and only if $q_{0} \in \hat{Q}_{s}$. 


\section{Conclusions}

The example given in appendix B highlights the advantages of the synthesis given here relative to the well-known supervisory synthesis of Ramadge (1989). The principal feature in favour of synthesis in by Ramadge (1989) is that the NTB specifications are given in terms of subsets of $[L(G)]^{\infty}$, which is more general than specifying i.o. visitations to a given Büchii state subset. However, the approach requires conversion of the NTB to finite behaviour using the prefix operator $\operatorname{pr}($.) and then synthesising the supervisor that meets the prefix specifications. The limitations of this method are as below.

- A supervisor having a finite state structre is possible only if $\operatorname{pr}(B)$ is regular (Ushio 1993).

- The synthesis requires that $B$ be closed relative to $[L(G)]^{\infty}$, which may be hard to obtain in practice (see appendix B). In case $B$ does not satisfy this constraint, the closed-loop can only be guaranteed to stay within $\vec{B}$, the topological closure of $B$ relative to $[L(G)]^{\infty}$, and not in $B$ which is the NTB specification (see appendix B).

The acceptable specifications used here, though relatively less general, are meaningful and adequate for a large CDEP class. Main advantages of our method, for example, are the following.

- The Büchii set specification is very meaningful in terms of closed -loop stability analysis (Özveren \& Willsky 1991). In fact, stability specifications in terms of Büchii sets is a very natural and logical extension of CDEPs based on finite state machine models (Eilenberg 1974). The synthesis then provides flexible stabilization of CDEPs.

- The method does not require construction of a grammar for acceptable NTB, thus reducing the computational complexity.

- It is easy to deal with, possibly conflicting, finite and non-terminating behaviour. This is due to the supervisor structure obtained and the operational flexibility achieved due to our switching function.

The supervisory synthesis approach as outlined above, therefore, presents a very efficient mechanism of ensuring flexible stabilization and satisfaction of other closed-loop specifications of CDEPs.

The authors are deeply grateful to one of the anonymous reviewers for pointing out a serious error in the proof of theorem 1 as well as other minor errors. They are also thankful to other reviewers for many helpful suggestions.

\section{Appendix A}

Completion of the proof of theorem 1 requires some auxillary definitions and lemmas. We begin by introducing the notion of a restricted supervisor. Let $G=\left(Q, \Sigma, \delta, q_{0}\right)$ be a CDEP. Suppose $\delta\left(q_{0}, s\right)=\dot{q} \in Q$ for some $s \in \Sigma^{*}$. Then $\dot{G}=(\dot{Q}, \Sigma, \dot{q}, \dot{\delta})$, where 
$\grave{Q} \subseteq Q$ is the set of states accessible from $\dot{q}$ and $\delta$ has the same action as that of $\delta$, is said to be a $s u b$-CDEP of $G$. A sub-CDEP, therefore, is part of the original CDEP that remains when we consider the system evolving from a later state. In the sequential context, the the Büchii set for the $s u b$-CDEP will be given by $Q_{m}^{\prime}=\dot{Q} \cap Q_{m}$.

Definition. Let $G$ be a CDEP and $G$ a $s u b$-CDEP of $G$. Suppose $T: Q \rightarrow \Gamma$ is a supervisor for $G$. Then $\dot{T}: \dot{Q} \longrightarrow \Gamma$ is said to be the restriction of $T$ to $\dot{Q}$ if:

$$
\dot{T}(q) \sigma=T(q) \sigma \quad \forall q \in \dot{Q}, \forall \sigma \in \Sigma .
$$

It follows directly from the above definition and the notions of non-blocking and regenerative supervision introduced earlier that if $T$ is a non-blocking and regenerative supervisor for $G$, so is $T$ for $G$. Next we explore the structure of $X_{i}, Q_{i}$ and $\hat{Q}((16)-(18))$ sets of $G$ when the Büchii set is restrictively altered.

Lemma Al. Let $G$ be a CDEP with $Q_{m}$ its associated Büchii set. Let $\bar{Q}_{m} \subseteq Q_{m}$, and let $\bar{Q}_{i}, \bar{X}_{i}$ and $\hat{\bar{Q}}$ be the control subsets of $G$ relative to Büchii set $\bar{Q}_{m}$. Then $\bar{Q}_{i} \subseteq Q_{i}, \forall i$.

Proof (By induction on $i$ ). At $i=0$, we have that $\bar{X}_{0}=\bar{Q}_{0}=\bar{Q}_{m}$. Now $Q_{0}=Q_{m} \Longrightarrow$ $\bar{Q}_{0} \subseteq Q_{0}$ since $\bar{Q}_{m} \subseteq Q_{m}$.

Assume that the hypothesis holds for $i=j$.

Now by definition (see (17)), we have that $q \in \bar{X}_{j+1} \Longrightarrow q \in\left(\tau \bar{Q}_{j} \cap \bar{Q}_{j}^{c}\right.$ ) and $\Sigma_{q, \bar{Q}_{j}^{c}} \subseteq$ $\Sigma_{c}$. Therefore $q \in \tau Q_{j} \cap\left(Q_{j}^{c} \cup\left(Q_{j}-\bar{Q}_{j}\right)\right)=\left(\tau Q_{j} \cap Q_{j}^{c}\right) \cup\left(\tau Q_{j} \cap\left(Q_{j}-\bar{Q}_{j}\right)\right)$ because $\bar{Q}_{j} \subseteq Q_{j}$ and $\bar{Q}_{j}^{c}=Q_{j}^{c} \cup\left(Q_{j}-\bar{Q}_{j}\right)$. Also note that $\Sigma_{q, \bar{Q}_{j}^{c}} \subseteq \Sigma_{c} \Longrightarrow \Sigma_{q, Q_{j}^{c}} \subseteq \Sigma_{c}$ since $Q_{j}^{c} \subseteq \bar{Q}_{j}^{c}$.

We, therefore, have that $\bar{X}_{j+1}=X_{j+1} \cup\left\{q \in \tau Q_{j} \cap\left(Q_{j}-\bar{Q}_{j}\right): \Sigma_{q, Q_{j}^{c}} \subseteq \Sigma_{c}\right\}$, where the second set is obviously in $Q_{j}$. Therefore, $\bar{X}_{j+1} \subseteq X_{j+1} \cup Q_{j}=Q_{j+1}$, and, consequently, $\bar{Q}_{j+1}=\bar{Q}_{j} \cup \bar{X}_{j+1} \subseteq Q_{j} \cup X_{j+1}=Q_{j+1}$. Hence $\bar{Q}_{j} \subseteq Q_{j}, \forall j \in N$.

Remark A1. It is obvious from the above proof that lemma $\mathrm{Al}$ holds if $G$ is replaced by a $s u b$-CDEP and the Büchii subset is compatibly chosen, as discussed earlier.

Next the proof of the necessity of theorem 1 follows:

Proof (Necessity of theorem 1). Let $G=\left(Q, \Sigma, \delta, q_{0}\right)$ be the given CDEP with $Q_{m}(\neq \Phi)$ as its associated Büchii state subset. Let $T$ be a non-blocking and regenerative supervisor for $G$.

We shall prove the necessity of the theorem 1 by induction on $\alpha(Q)$.

For $\alpha(Q)=1$, since $q_{0}=Q_{m}$, the result is obvious. Assume that the result holds for $\alpha(Q)=n$. Next, let $\alpha(Q)=n+1$. Suppose $\exists \sigma \in \Sigma$ such that $\delta\left(q_{0}, \sigma\right)=q_{0}$. Then if $\sigma \in \Sigma_{u}$, we shall have $q_{0} \in Q_{m}$, otherwise no regenerative supervisor can exist as the CDEP could indefinitely stay in $q_{0}$. If this were indeed the case then $q_{0} \in \hat{Q}$ and there is nothing further to prove. Therefore, without loss of any generality, we shall assume that in such a case $\sigma \in \Sigma_{c}$.

Next let $q_{1}, q_{2}, \cdots, q_{r} ; r \leq n$ be the states, other than $q_{0}$, which are 1-step accessible from $q_{0}$, i.e., $\forall i \leq r, q_{i}=\delta\left(q_{0}, \sigma_{i}\right)$ for some $\sigma_{i} \in \Sigma$. Consider the $s u b$-CDEPs $\dot{G}_{i}, 1 \leq$ 
$i \leq r$, generated from the states $q_{i}$, i.e., $\dot{G}_{i}=\left(\dot{Q}_{i}, \Sigma, q_{i}, \delta\right)$, where $\dot{Q}_{i} \subseteq Q$ is the state set accessible from $q_{i}$, and $\dot{Q}_{i m}=Q_{m} \cap \dot{Q}_{i}$ is the associated Büchii set. Since $\alpha\left(\dot{Q}_{i}\right) \leq n$, we have, from lemma $\mathrm{A} 1$ and remark $\mathrm{A} 1$, that, $\forall i, j ; i \leq r$ and $j \leq r_{i}, \dot{Q}_{i j} \subseteq Q_{j}$, i.e., the $j$ th control subset corresponding to the $i$ th $s u b$-CDEP is contained in the $j$ th control subset of $G$. Here $r_{i}$ denotes the length of the control subset chain upto its saturation (see (21)).

Furthermore, since the restricted supervisors $\dot{T}_{i}$ acting on $\dot{G}_{i}$ are non-blocking and regenerative, and since $\alpha\left(\dot{Q}_{i}\right) \leq n$, it follows by induction hypothesis that $q_{i} \in \hat{Q}_{i}(=$ $\dot{Q}_{i r_{i}}$, by (19)), $\forall i$. Next let $\hat{r}=\max _{i} r_{i}$. Then it follows that $\left\{q_{1}, q_{2}, \cdots, q_{r}\right\} \subseteq Q_{\hat{r}}$. Clearly then, $q_{0} \in \tau Q_{\hat{r}} \cap Q_{\hat{r}}^{c}$ and $\Sigma_{q_{0}, Q_{\hat{r}}^{c}} \subseteq \Sigma_{c}$ (since the only such transition can be to $q_{0}$ itself and that is controllable, by assumption); so that $q_{0} \in Q_{\hat{r}+1} \subseteq \hat{Q}$. This completes the proof.

\section{A simple synthesis example}

Consider the CDEP given below in the figure. Here $Q=\left\{q_{0}, q_{1}, q_{m}\right\}, \Sigma=\left\{\sigma_{1}, \sigma_{2}, \sigma_{3}, \sigma_{4}\right\}$ with $\Sigma_{c}=\left\{\sigma_{2}\right\}$ (say). Clearly $L(G)=\sigma_{1} \sigma_{2}^{*}\left(1+\sigma_{3} \sigma_{4}^{*}\right)$ and $[L(G)]^{\infty}=\left\{\sigma_{1} \sigma_{2}^{\omega}, \sigma_{1} \sigma_{2}^{*}\right.$ $\left.\sigma_{3} \sigma_{4}^{\omega}\right\}$. Let the acceptable (regenerative) non-terminating behaviour (NTB) be infinitely often i.o. visitations to the set $Q_{m} \doteq\left\{q_{m}\right\}$. The corresponding largest desired behaviour then is $B=\left\{\sigma_{1} \sigma_{2}^{*} \sigma_{3} \sigma_{4}^{\omega}\right\} \subseteq[L(G)]^{\infty}$. With this as the desired NTB, the synthesis procedure of $[R]$ gives a supervisor $M$, whose finite and NTB behaviour equals $L(G / M)=$ $\sigma_{1} \sigma_{2}^{*}\left(1+\sigma_{3} \sigma_{4}^{*}\right)$ and $[L(G / M)]^{\infty}=\left\{\sigma_{1} \sigma_{2}^{\omega}, \sigma_{1} \sigma_{2}^{*} \sigma_{3} \sigma_{4}^{\omega}\right\}$. The closed-loop NTB obtained is actually the (topological) closure (see $\S 2.1$ ) of $B$ relative to $[L(G)]^{\infty}$ and violates the specification of the desired NTB. This limitation of the procedure occurs because $B$ is not closed relative to $[L(G)]^{\infty}$.

If however we choose $B=\left\{\sigma_{1} \sigma_{3} \sigma_{4}^{\omega}\right\}$, which is closed relative to $[L(G)]^{\infty}$, as the desired NTB, then the algorithm of by Ramadge (1989) results in a supervisor $M$ such that $L(G / M)=\left\{\sigma_{1} \sigma_{3} \sigma_{4}^{*}\right\}$ and $[L(G / M)]^{\infty}=\left\{\sigma_{1} \sigma_{3} \sigma 4^{\omega}\right\}$ which satisfies the NTB specification. Note, however, that the resulting supervisor turns out to be very restrictive.

To obtain a less restrictive supervisor, we could choose $B_{k}=\left\{\sigma_{1} \sigma_{2}^{k} \sigma_{3} \sigma_{4}^{\omega}\right\}, k<$ $\infty$, which is closed w.r.t. $[L(G)]^{\infty}$. With this $B$ the synthesis by Ramadge (1989) results in a supervisor less restrictive than the earlier one (larger $k$ improves the performance), but the procedure requires construction of a grammar for $\operatorname{pr}\left(B_{k}\right)$ for every desired $k$.

Using the algorithm of $\S 3$, we obtain the following contol subsets as follows.

$$
X_{0}=Q_{m}=\left\{q_{m}\right\} ; X_{1}=\left\{q_{1}\right\} ; X_{2}=\left\{q_{0}\right\} . \hat{Q}_{s}=Q ; \hat{Q}_{m_{s}}=Q_{m} \text {. }
$$

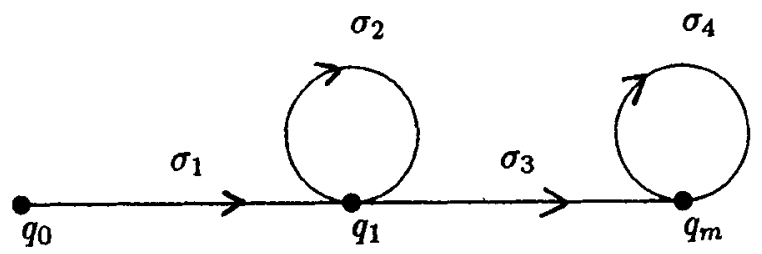

Figure B1. A CDEP example. 
Observe that $q_{0} \in \hat{Q}_{s}$. We, therefore, get:

The supervisor $R \doteq R(q) \sigma=0$ if $q=q_{1}$ and $\sigma=\sigma_{2}$; and $R(q) \sigma=1$ otherwise.

The supervisor $S \doteq S(q) \sigma=1 \forall q \in Q$ and $\sigma \in \Sigma$.

The NTB of the closed-loop under the composite supervisor $T$ is then equal to $B_{k}=$ $\left\{\sigma_{1} \sigma_{2}^{k} \sigma_{3} \sigma_{4}^{\omega}\right\}$ which is the desired NTB. The synthesis, however, avoids grammar construction as well as specification of the language that guarantees the desired regenerative behaviour, e.g. $\operatorname{pr}(B)$, which is necessary for other approaches. The parameter $k$ here, in fact, parametrises the family of non-blocking regenerative supervisors for the system in the example given.

\section{References}

Brave Y, Heyman M 1989 On stabilization of discrete event processes. In IEEE Proceedings of 28th Conference on Decision and Control, Tampa, FL, pp 2737-2742

Eilenberg S 1974 Automata, languages and machines (New York: Academic Press) vol. A

Hopcroft J E, Ullman J D 1979 Introduction to automata theory, languages and computation (Reading, MA: Addison-Wesley)

Özveren C M, Willsky A S 1991 Stability and stabilizability of discrete event dynamic systems. J. ACM 38: 730-752

Ramadge P J 1989 Some tractable supervisory control problems for discrete event systems described by Büchii automata. IEEE Trans. Autom. Control 34: 10-19

Kumar R, Garg V, Marcus S I 1992 On supervisory control of sequential behaviours. IEEE Trans. Autom. Control 37: 1978-1984

Kumar R, Garg V, Marcus S I 1993 Predicates and predicate transformers for supervisory control of discrete event dynamic systems. IEEE Trans. Autom. Control 38: 232-247

Kumar R, Garg V, Marcus S I 1993 Language stability and stabilizability of discrete event dynamical systems. SIAM J. Control Optimization 31: 1294-1320

Ramadge P J, Wonham W M 1987 Supervisory control of a class of discrete event processes. SIAM J. Control Optimization 25: 206-230

Ushio T 1993 A necessary and sufficient condition for the existence of finite state supervisors for discrete event systems. IEEE Trans. Autom. Control 38: 135-137

Valavi A G 1994 A theory for regenerative supervision of discrete event processes. M Tech thesis, Indian Institute of Technology, Kanpur

Wonham W M, Ramadge P J 1987 On the supremal controllable sublanguage of a given language. SIAM J. Control Optimization 25: 637-659 